\title{
Targeting type I interferon-mediated activation restores immune function in chronic HIV infection
}

\author{
Anjie Zhen, ${ }^{1}$ Valerie Rezek, ${ }^{1}$ Cindy Youn, ${ }^{1}$ Brianna Lam, ${ }^{1}$ Nelson Chang, ${ }^{1}$ Jonathan Rick, ${ }^{1}$ Mayra Carrillo, ${ }^{1}$ Heather Martin, ${ }^{1}$ \\ Saro Kasparian, ${ }^{1}$ Philip Syed, ${ }^{1}$ Nicholas Rice, ${ }^{1}$ David G. Brooks, ${ }^{2,3}$ and Scott G. Kitchen' \\ 'Division of Hematology and Oncology, Department of Medicine, UCLA AIDS Institute, David Geffen School of Medicine, UCLA, Los Angeles, California, USA. PPrincess Margaret Cancer Center, \\ Immune Therapy Program, University Health Network, Toronto, Ontario, Canada. ${ }^{3}$ Department of Immunology, University of Toronto, Toronto, Ontario, Canada.
}

\begin{abstract}
Chronic immune activation, immunosuppression, and T cell exhaustion are hallmarks of HIV infection, yet the mechanisms driving these processes are unclear. Chronic activation can be a driving force in immune exhaustion, and type I interferons (IFN-I) are emerging as critical components underlying ongoing activation in HIV infection. Here, we have tested the effect of blocking IFN-I signaling on T cell responses and virus replication in a murine model of chronic HIV infection. Using HIVinfected humanized mice, we demonstrated that in vivo blockade of IFN-I signaling during chronic HIV infection diminished HIV-driven immune activation, decreased T cell exhaustion marker expression, restored HIV-specific CD8 T cell function, and led to decreased viral replication. Antiretroviral therapy (ART) in combination with IFN-I blockade accelerated viral suppression, further decreased viral loads, and reduced the persistently infected HIV reservoir compared with ART treatment alone. Our data suggest that blocking IFN-I signaling in conjunction with ART treatment can restore immune function and may reduce viral reservoirs during chronic HIV infection, providing validation for IFN-I blockade as a potential therapy for HIV infection.
\end{abstract}

\section{Introduction}

The HIV-specific T cell response is critical to control HIV replication following infection $(1,2)$. However, for a variety of reasons, including the insufficient generation and maintenance of functional $\mathrm{T}$ cell responses, $\mathrm{T}$ cells are incapable of clearing the infection $(3,4)$. T cell dysfunction due to cellular exhaustion arises during many chronic infections in response to ongoing antigen stimulation, which results in the expression of immunosuppressive factors including PD-1, TIM-3, and IL-10 (5-8). In addition to immunosuppressive mechanisms, chronic inflammation and immune activation may further foster $\mathrm{T}$ cell exhaustion, limiting control of infection $(9,10)$. In fact, chronic immune activation is a hallmark of HIV infection, and higher levels of activated $\mathrm{CD}^{+}$and $\mathrm{CD} 8^{+} \mathrm{T}$ cells are directly correlated with accelerated disease progression (11-14). Yet, the mechanisms underlying the emergence of $\mathrm{T}$ cell exhaustion and global immune activation due to chronic HIV infection have yet to be defined.

Accumulating evidence suggests that chronic type I interferon (IFN-I) signaling enhances immune activation and drives the expression of multiple inhibitory factors that inhibit antiviral immunity and contribute to viral persistence $(5,10,15-17)$. IFN-I is critical for the initiation of antiviral responses, and blockade of IFN-I signaling during acute SIV infection in rhesus macaques resulted in a loss of antiviral control culminating in increased SIV replication, enhanced CD4 T cell depletion, and accelerated disease progression $(15,18)$. Conversely, the same study also found that treatment with recombinant IFN- $\alpha$ (IFN- $\alpha 2 a)$ led to

\section{Related Commentary: p. 103}

Conflict of interest: The authors have declared that no conflict of interest exists. Submitted: July 13, 2016; Accepted: October 27, 2016.

Reference information: / Clin Invest. 2017;127(1):260-268. doi:10.1172/JCI89488. an IFN-desensitized state and accelerated disease progression despite initial heightened resistance to infection $(15,18,19)$. Previous clinical trials treating HIV patients with pegylated IFN- $\alpha 2$ a exhibited limited antiviral effects and no correlation between viral load decrease and plasma IFN- $\alpha 2$ a concentration $(15,19-21)$. These results highlight the complex roles that IFN-I plays during antiviral responses. Importantly, the effects of IFN-I signaling on $\mathrm{T}$ cell responses and HIV replication, once persistent infection is established, at a time when the antiviral roles of IFN-I have been suggested to be blunted $(22,23)$, remain unclear.

\section{Results}

Expression of exhaustion and activation markers is elevated in $T$ cells in chronically infected humanized BLT mice. Cellular immune responses in HIV-infected NOD SCID common $\gamma$ chain-deficient (NSG)/humanized bone marrow-human fetal liver and thymus (BLT) mice (NSG-BLT mice) have been recently demonstrated to closely mirror those in humans $(4,24-30)$, making it a good model to study HIV immune pathology and immune-based therapies in vivo. To investigate immune activation and immune exhaustion in vivo, we generated NSG-BLT mice (for representative flow plots of human lymphocytes, see Supplemental Figure 1; supplemental material available online with this article; doi:10.1172/ JCI89488DS1) and mock-infected or infected mice with HIV-1 and followed the expression of the immune inhibitory receptors PD- 1 and TIM-3 and the activation markers HLA-DR and CD38 on $\mathrm{CD}^{+}$and $\mathrm{CD} 4^{+} \mathrm{T}$ cells (for representative staining of the markers, see Supplemental Figure 2). During chronic HIV infection (9-13 weeks), HLA-DR, CD38, PD-1, and TIM-3 were all elevated on human $\mathrm{T}$ cells, particularly on human $\mathrm{CD}^{+} \mathrm{T}$ cells, compared with $\mathrm{T}$ cells from uninfected mice generated from the same donor tissues (Figure 1, A-D, and Supplemental Figure 2A), indicating 
ongoing $\mathrm{CD}^{+} \mathrm{T}$ cell exhaustion and immune activation in the NSG-BLT model. In addition, we found that expression of TIM-3 gradually increased on T cells throughout HIV infection (Supplemental Figure 2C). Similar to previous reports in HIV-infected patients $(1,2)$ and lymphocytic choriomeningitis virus (LCMV) -infected mice, in HIV-infected NSG-BLT mice we found subsets of $\mathrm{CD}^{+} \mathrm{T}$ cells that overexpressed both PD- 1 and TIM- 3 exhaustion markers $(3,4)$, and they displayed significantly reduced ability to produce proinflammatory cytokines upon mitogen stimulation, suggesting impaired functions of these T cells (Figure 1, E and F).

IFN-I signaling is chronically elevated during HIV-1 infection. Chronic IFN-I signaling is strongly associated with immune activation and suppression in persistent virus infections (5-8). To examine the extent of IFN-I signaling in vivo, we longitudinally measured HIV RNA expression and the expression of the IFN-Iinduced signature genes (ISGs) MX1 and OAS1 in both infected and uninfected mice. Humanized BLT mice support sustained HIV replication 7 weeks after infection (Figure 2A and refs. 9, 10) with gradual loss of CD4 cells and decreased CD4/CD8 ratios in the peripheral blood (Figure 2, B and C). MX1 expression levels in the peripheral blood increased rapidly, and both $M X 1$ and OAS1 remained elevated in the blood and tissue throughout HIV infection (Figure 2, D and E) in comparison with uninfected mice. Thus, IFN-I-induced gene expression is maintained in multiple compartments following HIV infection in NSG-BLT mice.

IFN-I receptor blockade effectively lowers ISG expression and reverse immune exhaustion. To investigate the impact of chronic IFN-I signaling on inflammation and T cell exhaustion, HIVinfected BLT mice were treated beginning 13 weeks after infection with an anti-human IFN receptor 2 (IFNR2) blocking antibody or an isotype control antibody for 1 week. IFNR blockade lowered gene expression of the ISGs MX1,OAS1, and IRF7 (Figure 3A and Supplemental Figure $5 \mathrm{~A}$ ) in the peripheral blood, demonstrating that anti-IFNR treatment blocked human IFN-I signaling in vivo. IFNR blockade did not affect overall $\mathrm{CD}^{+} \mathrm{T}$ cell percentages among $\mathrm{T}$ cells, or CD4/CD8 $\mathrm{T}$ cell ratios or CD4 and CD8 $\mathrm{T}$ cell numbers in the peripheral blood (Supplemental Figure 3, A-D). IFNR blockade, but not the isotype antibody, significantly reduced levels of PD-1, TIM-3, and CD38 expression on CD8 T cells (Figure 3B, Supplemental Figure 4, and Supplemental Figure 5, B-E). Further, $\mathrm{CD}^{+} \mathrm{T}$ cells from IFNR-blocked mice produced higher levels of IFN- $\gamma$ and IL-2 following mitogen PMA/ionomycin stimulation (Figure 3, C and D). To investigate whether HIV-specific CD8 T cell responses were improved, splenocytes from HIV-1-infected, isotype antibody-treated, or anti-IFNR2 antibody-treated mice were stimulated with an HIV clade B peptide pool (Gag, Env, Nef, and Pol) (11-14). IFNR blockade treatment elevated the percentage and number of HIV-specific IFN- $\gamma$-producing $\mathrm{CD} 8^{+} \mathrm{T}$ cells (Figure 3, E-G) as well as increasing the expression of IFN- $\gamma$ produced by single HIV-specific cells (Figure $3 \mathrm{H}$ ), indicating functional restoration of the HIV-specific T cell response when IFN-I signaling is alleviated. Interestingly, we also observed a reduction of HLA-DR on $\mathrm{CD}^{+}{ }^{+} \mathrm{T}$ cells following IFNR blockade (Figure 3I), indicating a reduction in $\mathrm{CD} 4^{+} \mathrm{T}$ cell activation.

IFNR blockade effectively reduces viral load. With the decreased expression of exhaustion factors and increased levels of IFN- $\gamma$ producing HIV-specific $\mathrm{CD}^{+} \mathrm{T}$ cells, as well as reduction in $\mathrm{CD} 4^{+}$
$\mathrm{T}$ cell activation, we then measured whether such improvement in CD8 $\mathrm{T}$ cell responses and overall immune activation reduction could lead to change in viral load. Interestingly, we found that IFNR blockade, but not isotype treatment, led to a reduction in virus loads when administered during the chronic stage of infection (Figure 4A).

Concurrent IFNR blockade and antiretroviral therapy result in faster viral suppression. In all, blocking IFN-I signaling during persistent viral infection can have multiple beneficial effects on immune responses and can lead to decreased HIV replication. To investigate the impact of blocking IFN-I signaling in conjunction with combination antiretroviral therapy (ART), humanized BLT mice were infected with $\mathrm{HIV}_{\mathrm{NL4-3HSA}}$, which is a replication-competent virus that contains a cell surface-expressed reporter gene (murine heat stable antigen [HSA]) whose detection is indicative of viral expression $(5,10,15-17)$. Importantly, HIV $_{\text {NL4-3HSA }}$ replicates with kinetics and titers analogous to those of the parental $\mathrm{HIV}_{\mathrm{NL4}-3}$ in BLT mice (15, 18). Eight weeks after HIV infection, mice were treated with a daily regimen of ART (tenofovir disoproxil fumarate [TDF]/ Emtricitabine [FTC]/raltegravir) for 3 weeks $(n=9)$, and another set of mice were treated with daily ART for 3 weeks including 1 week of concurrent IFNR blockade during ART treatment $(n=6)$. ART treatment reduced IFN-I signaling, and the combination of ART treatment with IFNR blockade resulted in a greater reduction in IFN-I signaling as shown by decreased expression of the ISGs MX1 and OAS1 (Figure 4B). Combined treatment of mice with ART and IFNR blockade also further reduced TIM-3 expression on CD8 cells to levels similar to those in uninfected controls (Figure 3C). When stimulated with PMA/ ionomycin, treatment with either IFNR blockade or ART alone restored CD8 production of cytokines, and CD8 cells from mice cotreated with ART and IFNR blockade produced a further improved level of cytokines that was similar to that in uninfected controls (Figure 3D). This suggests an additive benefit of ART and IFN-I blockade. ART treatment alone resulted in significant reduction of viral loads within 3 weeks of treatment compared with untreated infection (Figure 4E). Interestingly, blocking IFNR for 1 week in combination with ART led to a significantly greater reduction in HIV viral loads compared with IFNR blockade or ART alone (Figure 4, E and F, which shows viral load fold change after each treatment). This suggests that concurrent IFNR blockade during ART treatment of chronic HIV infection is beneficial to accelerate control of the virus.

Concurrent IFNR blockade and ART reduce levels of reactivatable viral reservoirs. The persistently infected, activation-inducible HIV reservoir represents a major obstacle to eradication. To determine how IFNR blockade affects the activation-inducible HIV reservoir, splenocytes were harvested from HIV NL4-3HAA -infected mice treated with isotype antibody, anti-IFNR blocking antibody, ART, or ART with anti-IFNR blocking antibody. HIV reporter gene-expressing (i.e., productively infected) cells were removed by sorting, leaving only cells not expressing virus $(15,18,19)$. We then measured HIV DNA copies from half of these cells and reactivated the other half with anti-CD3/anti-CD28 stimulation, in the presence of the protease inhibitor indinavir to prevent subsequent viral infection and spread in the culture. After 3 days, supernatant from the reactivation culture was collected for p24 ELISA assay to measure the production of viral particles and HIV RNA expression $(15,19-21)$ (for schematic outline of the viral reactivation assay, see Supplemental 
A
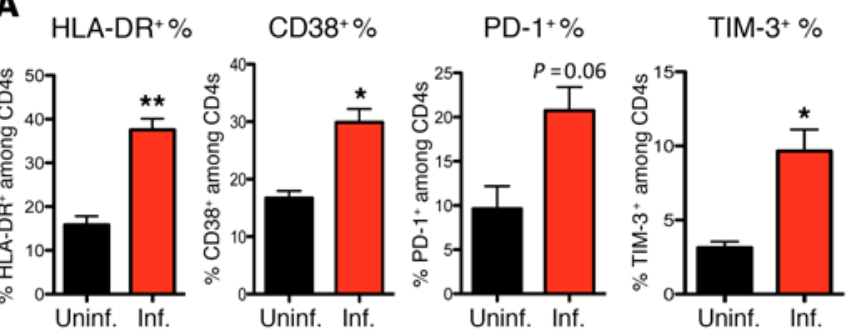

C

HLA-DR $+\%$

$\mathrm{CD} 38^{+} \%$
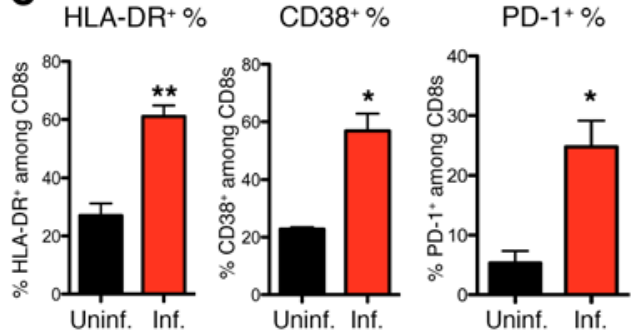

TIM-3+\%

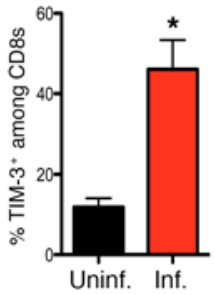

B
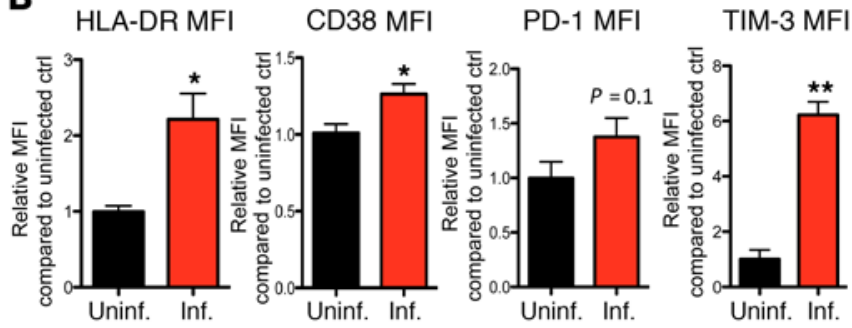

D

HLA-DR MF

CD38 MFI

PD-1 MFI

TIM-3 MFI
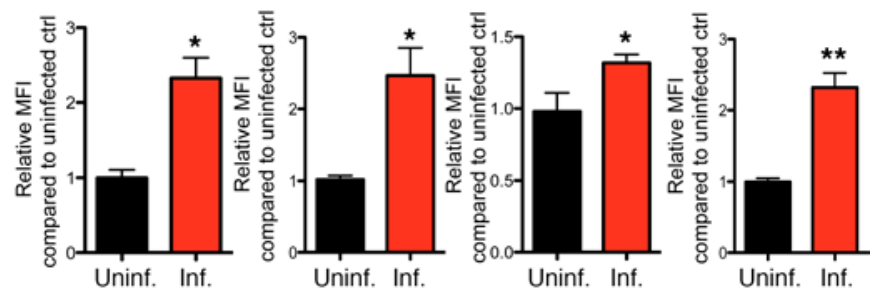

E
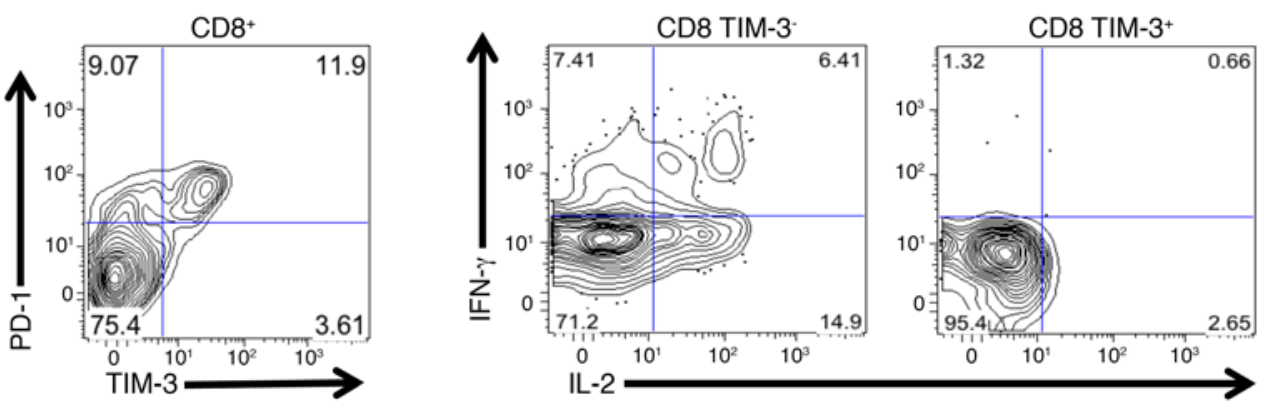

$\mathbf{F}$
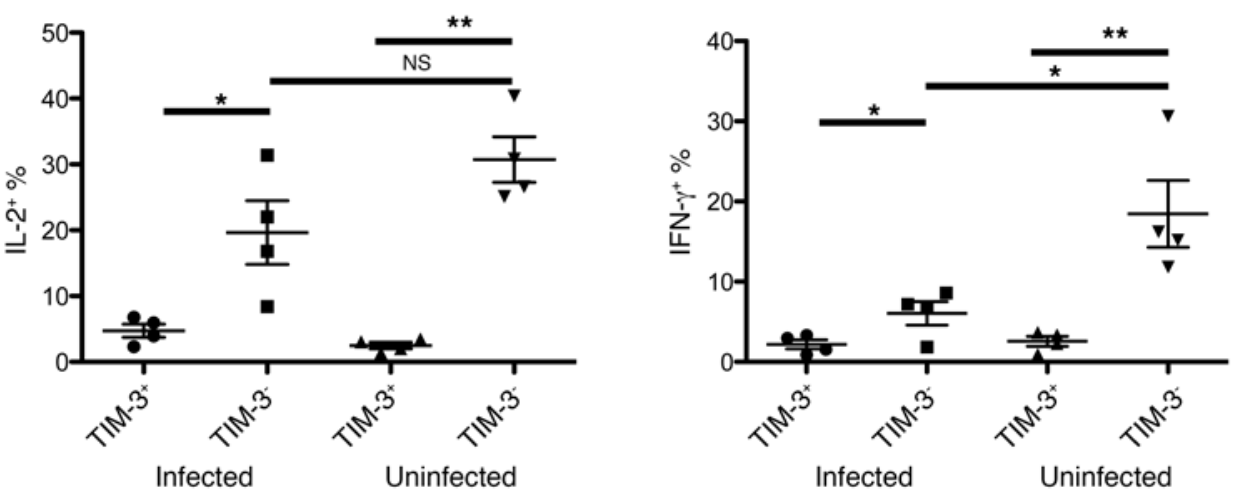

Figure 1. Chronic HIV infection results in elevated expression of activation and exhaustion markers and exhaustion of viral-specific CD8 cells. NSG-BLT humanized mice were constructed by implantation of fetal liver and fetal thymus as well as hematopoietic stem cells into the NSG mice. After human immune reconstitution, mice were mock-infected or infected with HIV $_{\mathrm{NL4-3}}$. Thirteen weeks after infection, whole blood from each mouse was collected, and cells were stained with anti-human antibodies CD45, CD3, CD4, CD8, TIM-3, PD-1, and HLA-DR and analyzed by flow cytometry. (A-D) Expression of HLA-DR, CD38, PD-1, and TIM-3 on human CD4 (A and $\mathbf{B})$ and CD8 cells (C and $\mathbf{D})$ was accessed by gating and by measurement of the percentage positive $(\mathbf{A}$ and $\mathbf{C})$ and relative MFI (B and $\mathbf{D}$ ) of marker expression ( $n=3-5$ mice per group and more than 1,000 events were acquired for each flow analysis). For relative MFI, data displayed are the mean relative MFI \pm SEM of these markers as compared with the mean MFI of uninfected controls, showing differences between these populations. (E) Splenocytes from HIV-infected NSG mice were stimulated with mitogen for 6 hours with GolgiPlug and were stained for expression of CD8, TIM-3, PD-1, and intracellular expression of IL-2 and IFN- $\gamma$. More than 1,000 events were acquired for each flow analysis, and the experiment was repeated more than 3 times. (F) Summary and statistical analysis of the intracellular cytokine expression assay in $\mathbf{E}(n=4-6$ mice per group). The Mann-Whitney test was used to compare 2 groups $\left({ }^{*} P<0.05,{ }^{* *} P<0.005\right)(\mathbf{A}-\mathbf{E})$, and the Kruskal-Wallis test was used for multiple comparisons $(\mathbf{F}, P<0.05)$. Data represent mean \pm SEM. 
A

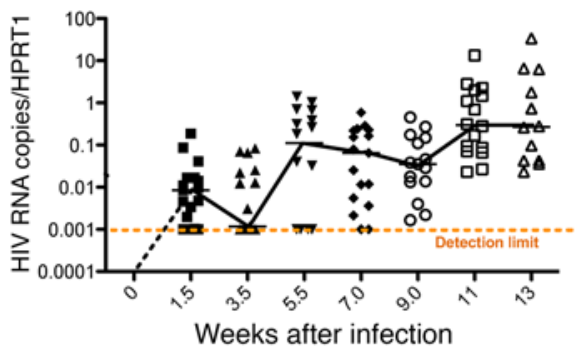

C

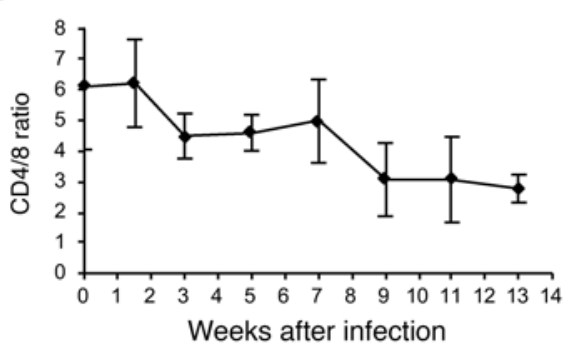

E

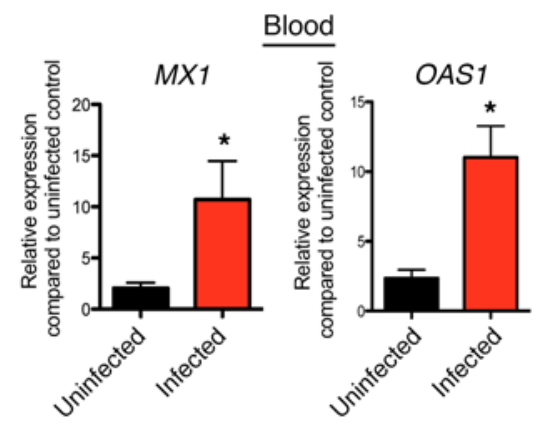

B

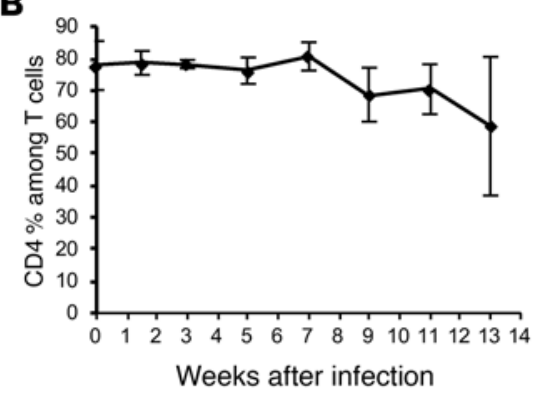

D
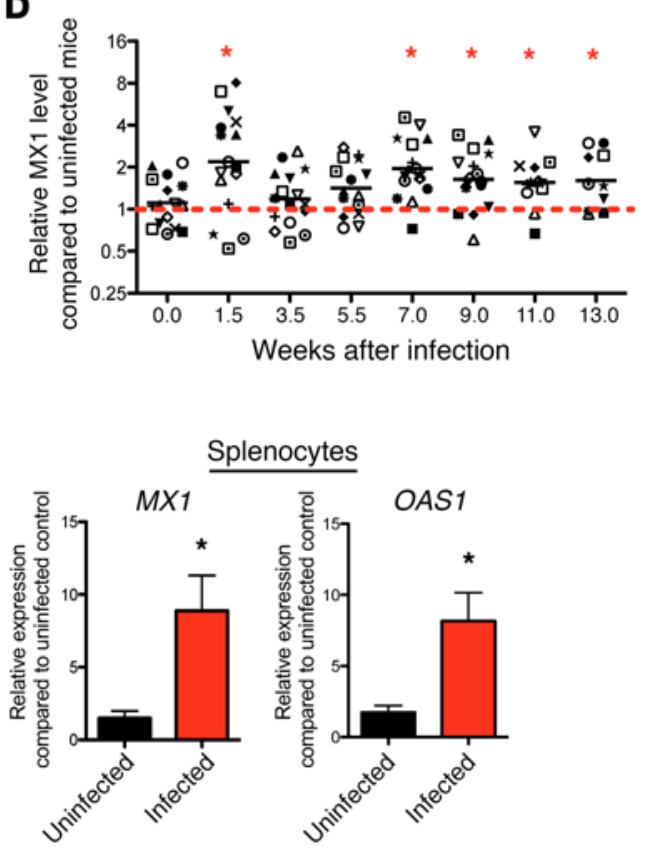

Figure 2. IFN-I signaling is chronically elevated during HIV-1 infection. (A) Change of HIV cell-associated RNA levels in peripheral blood mononuclear cells (PBMCs) from BLT mice after HIV infection as compared with expression of the human housekeeping gene hypoxanthine phosphoribosyltransferase 1 (HPRT). Detection limit is shown by dashed orange line $(n=12-20)$. (B) CD4\% among T cells in PBMCs from BLT mice after HIV infection $(n=12-20$ per group). CD4\%, percent of CD4+ cells among T cells in PBMCs. (C) CD4/ CD8 ratio of T cells in PBMCs from BLT mice after HIV infection ( $n=12-20$ per group). (D) Expression levels of the $M X 1$ gene in PBMCs throughout chronic HIV-1 infection in comparison with uninfected animals ( $n=11-20$ per group). Red dashed line defines the relative levels of MX1 gene expression in uninfected mice, which is equal to 1. (E) Expression level of MX1 and OAS1 in PBMCs and splenocytes from uninfected and infected mice as measured by reverse transcriptase PCR (RT-PCR) ( $n=4-16$ per group). ${ }^{*} P<0.05$, Mann-Whitney $U$ test. Data represent mean \pm SEM.
Figure 6A). As shown in Figure 4G, without CD3/CD28 stimulation, very little viral production was detected, indicating that sorting effectively eliminated productively infected cells. Cells from mice treated with IFNR blockade demonstrated reduced levels of inducible virus following stimulation, similar to those observed in ART-treated mice; both were less than those seen in non-ART/ isotype treatment (Figure 4G). Similarly, we observed significant reduction of HIV RNA production from stimulated $\mathrm{HSA}^{-}$cells from mice that received IFN-I blockade as compared with isotype control (Supplemental Figure 6B). ART treatment further lowered the HIV RNA, and combined treatment of ART and IFNR blockade had the lowest HIV RNA production, again indicating a smaller HIV reservoir. We also observed a trend of decreased HIV DNA in unstimulated $\mathrm{HSA}^{-}$cells from IFNR blockade-treated mice versus isotype-treated mice (Supplemental Figure 6C). However, it did not reach statistical significance. It may be due to the fact that defective provirus rapidly accumulates during active HIV-1 infection and measurement of HIV DNA may not reflect replication-competent HIV $(22,23)$. Nonetheless, we observed significantly reduced HIV DNA levels in ART- and IFNR blockade-treated mice as compared with mice treated only with ART (Supplemental Figure 6C). All together, these results indicate accelerated reduction of the amount of reactivatable virus to background levels when IFNR is therapeutically inhibited in conjunction with ART.

\section{Discussion}

One of the hallmarks of HIV infection is chronic immune activation that promotes viral replication and CD4 depletion. In addition, chronic immune activation leads to the upregulation of inhibitory molecules and is widely believed to be one of the driving forces of immune exhaustion, in which effector cells lose their function and proliferation capacities $(4,24-30)$. Here we show, for the first time to our knowledge, that activation and exhaustion markers such as HLA-DR, CD38, PD-1, and TIM-3 are elevated in humanized BLT mice chronically infected with HIV-1 (Figure 1A) and that CD8 T cells expressing multiple suppressive receptors such as PD-1 and TIM-3 define an exhausted effector T cell population (Figure 1B) as observed in human HIV patients $(1,14)$ and mice chronically infected with LCMV (3). In addition, as in HIV-infected patients, we observed upregulated ISGs $M X 1$ and $O A S 1$ in chronically infected BLT mice compared with uninfected mice (Figure 2). These results indicate that NSG-BLT mice infected with HIV can mirror immune activation and immune exhaustion observed in HIV-infected patients, making them an important animal model to study HIV immunopathogenesis and immunotherapy.

Given the complex activities of IFN-I during acute and chronic viral infection $(18,23)$, it is crucial to delineate the effect of IFN-I signaling during different phases of HIV infection, as improper modulation of anti-IFN or IFN- $\alpha$ treatment during acute infection 
A

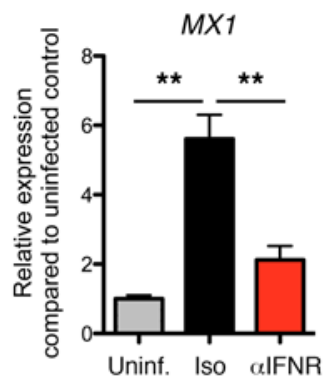

B
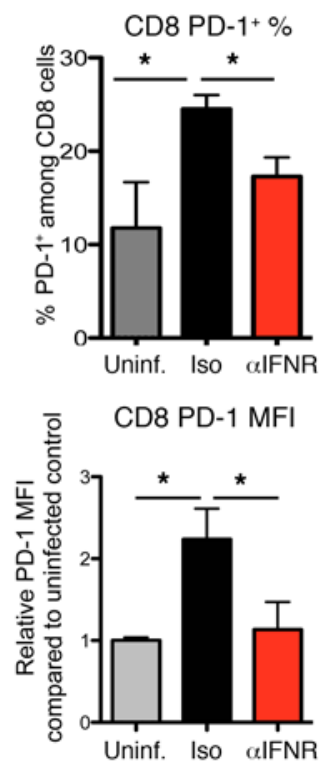

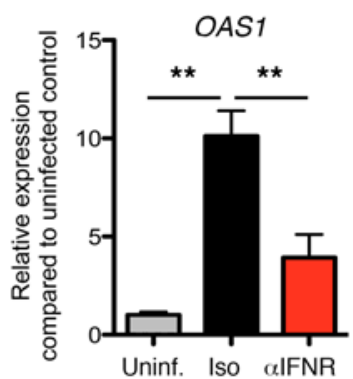

CD8 TIM $-3^{+} \%$
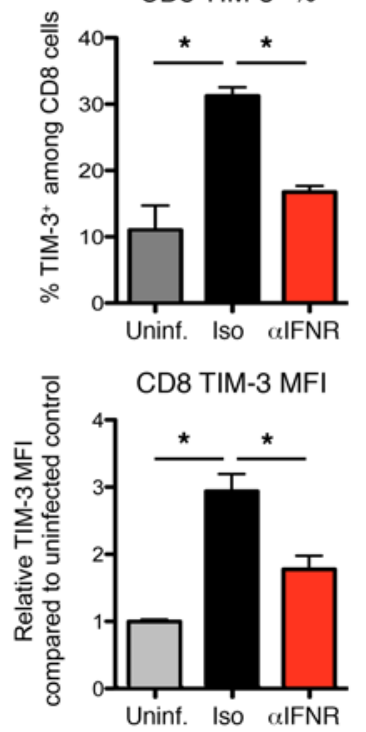

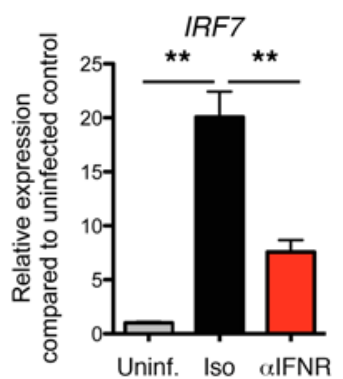

$\mathrm{CD} 8 \mathrm{CD} 38^{+} \%$
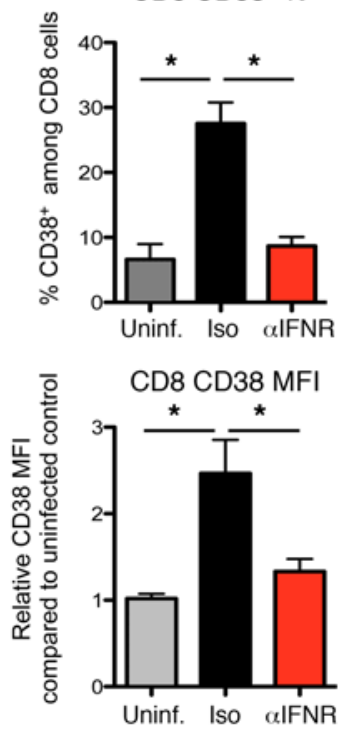

C

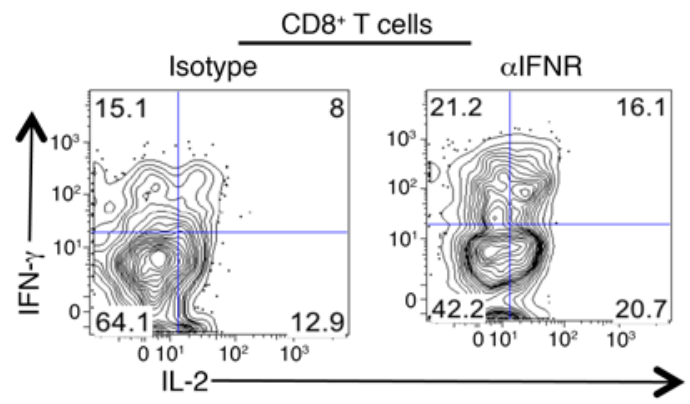

D

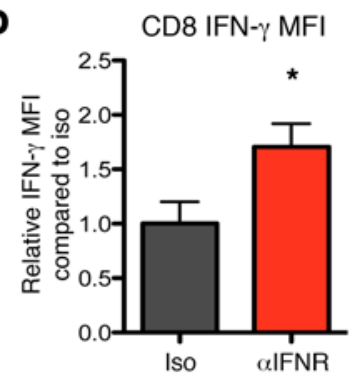

CD8 IL-2 MFI

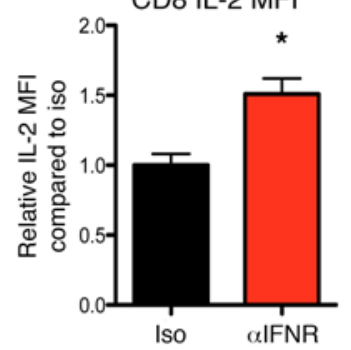

E

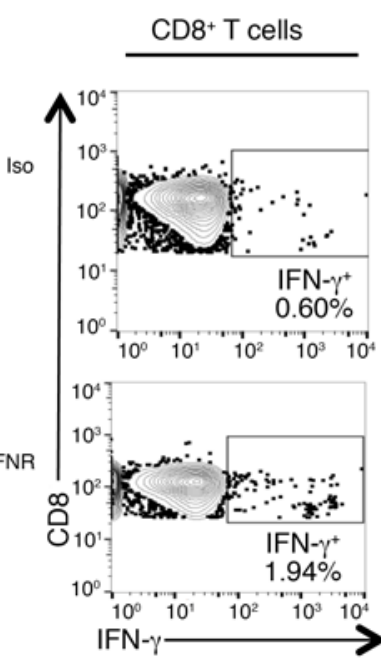

F $\quad$ CD8 IFN- $\gamma^{+} \%$

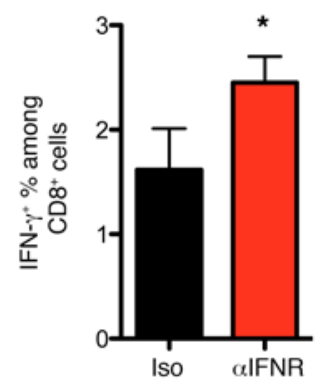

G No. of CD8 IFN- $\gamma^{+}$
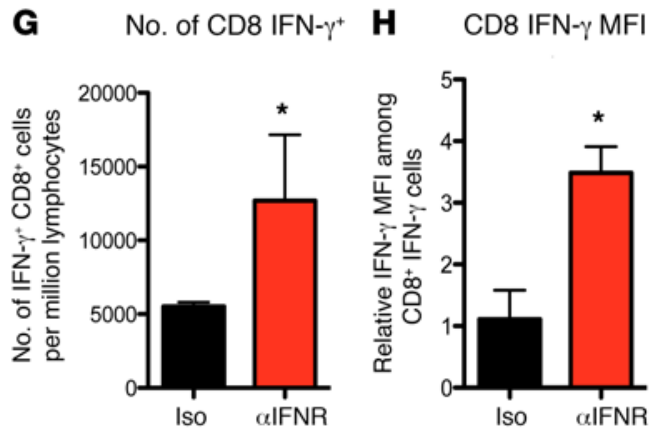

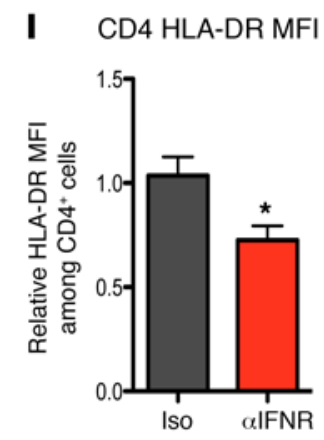


Figure 3. IFNR blockade effectively lowers ISG expression levels and reverses immune exhaustion. (A) Twelve weeks after infection, mice were treated with IFNR blocking antibody ( $\alpha$ IFNR) or an isotype antibody control (iso) for 8 days. Expression levels of the ISGs MX1, OAS1, and IRF7 in human PBMCs from humanized BLT mice after treatment were measured by real-time RT-PCR ( $n=3-5$ per group). (B) PD-1, TIM-3, and CD38 expression as measured by flow cytometry (quantitatively by gating of percentages positive and relative MFIs \pm SEM) on T cells from uninfected or infected mice that were treated with IFNR blocking antibody or isotype control ( $n=3-7$ per group). (C) Splenocytes from HIV-1-infected, isotype antibody-treated, or IFNR antibody-treated mice were stimulated with PMA/ionomycin for 6 hours, and IFN- $\gamma$ and IL-2 production by CD8 cells was measured by flow cytometry (representative of $n=3$ per group). (D) Summary of $\mathbf{C}$ as measured by relative MFI. (E) Splenocytes from HIV-1-infected, isotype-treated, or IFNR antibody-treated mice were stimulated with an HIV-1 clade B peptide pool (Pol, Gag, Env, and Nef), and production of IFN- $\gamma$ by CD8 cells was measured by flow cytometry (representative of $n=3$ per group). (F) Summary of the total percentage of IFN- $\gamma^{+}$CD8 ${ }^{+}$cells after HIV peptide pool stimulation. (C) Summary of IFN- $\gamma$ production by CD8 ${ }^{+}$cells as measured by relative MFI after HIV peptide pool stimulation. (H) Summary of IFN- $\gamma^{+}$CD8 ${ }^{+}$cell numbers per million lymphocytes. (I) Summary of the relative HLA-DR MFI on CD4 T cells from infected mice treated with isotype or type I IFNR antibody ( $n=4-6$ per group). The Mann-Whitney test was used to compare 2 groups $\left({ }^{*} P<0.05,{ }^{* *} P<0.005\right)$, and the Kruskal-Wallis test was used for multiple comparisons ( $\mathbf{A}$ and $\mathbf{B}$, both $P<0.05$ ). Data represent mean $\pm \mathrm{SEM}$. Representative experiments were performed more than 3 times (C and $\mathbf{E})$.

could have drastic effects, leading to both faster viral replication and disease progression $(5,7,17,18)$. Our results demonstrate that IFN-I signaling during chronic HIV infection is one of the driving forces underlying chronic inflammation, immune activation, and $\mathrm{CD} 8^{+} \mathrm{T}$ cell exhaustion and that blockade of IFN-I signaling could potentially improve $\mathrm{T}$ cell immune responses. Contrary to what was observed at the onset of acute SIV infection (18), where IFNR antagonist resulted in loss of viral control and faster disease progression, we found that IFNR blockade during the chronic stage of the HIV infection led to a reduction of immune activation, enhanced HIV-specific $\mathrm{CD} 8^{+} \mathrm{T}$ cell function, and decreased HIV plasma viral load. In addition, we observed a reduction in CD4 T cell activation upon IFNR blockade, indicating that a chronically elevated level of IFN-I may have directly or indirectly contributed to the systematic activation of $\mathrm{CD} 4 \mathrm{~T}$ cells. The reduced $\mathrm{CD} 4^{+} \mathrm{T}$ cell activation may have slowed the dissemination of the virus and in combination with enhanced immune competence contributed to the decrease of viral load. Furthermore, we demonstrate that concurrent ART and IFNR blockade during chronic HIV infection accelerated viral suppression and ultimate reduction in the reactivatable HIV reservoir. The reduction of this reservoir may have resulted from better viral control or a release of viral expression that is suppressed by IFN-I responses. IFN-I signaling has been shown to suppress viral transcription in HIV-infected cells (31), and blockade of IFN-I signaling in the presence of ART treatment may reduce the establishment of persistently infected reservoirs. Our study highlights the multifaceted activities of IFN-I during viral infection and suggests that it may act as a double-edged sword during chronic HIV infection, fueling immune activation and viral dissemination. Further studies are needed to delineate the role of IFN-I signaling during persistent HIV infection and validate IFNR blockade's therapeutic potential for treating HIV infection, particularly in conjunction with ART.

\section{Methods}

Humanized BLT mice. Humanized mice were constructed as described previously $(15,30)$. In short, $\mathrm{CD} 34^{+}$cells were purified via magnetic cell sorting with CD34 microbeads (Miltenyi Biotec) from freshly obtained fetal liver tissues. NOD-SCID-common $\gamma$ chain knockout $\left(\mathrm{c}_{\gamma}^{--}\right)$mice (NSG mice) mice were sublethally irradiated (2.7 Gy) before the surgery and implanted with fetal liver and thymus derived from the same donor as the $\mathrm{CD}_{3} 4^{+}$cells. Afterward, mice were injected with 0.5 million to 1 million $\mathrm{CD} 34^{+}$cells. After 8-10 weeks, each mouse was bled retro-orbitally to check human immune cell engraftment. Each donor tissue can be used to construct 15-25 mice. Mice that had more than $50 \%$ human lymphocytes in the peripheral blood mononuclear cells were used for HIV infection and further experiments. A standard healthy uninfected humanized BLT mouse contains 0.5 million to 2 million human lymphocytes per milliliter in the blood and 10 million to 30 million human lymphocytes per milliliter in the spleen.)

Antibodies and flow cytometry. The following antibodies were used in flow cytometry: CD45 (clone HI30), CD3 (clone OKT3), CD4 (clone RPA-T4), CD8 (clone SK1), CD38 (clone HIT2), HLADR (clone L240), PD-1 (clone ebioJ105) (eBioscience), TIM-3 (clone F38-2E2) (Biolegend), and anti-HIV-1 core antigen clone KC57 (Beckman Coulter). Antibody for cell surface markers were conjugated to FITC, PE, PerCP-Cy5.5, PE-Cy5, PE-Cy7, EVD, APC, APC-eFluor 780, Alexa Fluor 700, eFluor 405, Pacific Orange, or Pacific Blue in appropriate combination. The cells were acquired using an LSRFortessa flow cytometer and FACSDiva software (BD Biosciences). Data were analyzed using FlowJo software. At least 1,000 cells were acquired for each analysis, and each representative flow plot was repeated more than 3 times.

In vitro cytokine assay. We assessed purified splenocytes from BLT mice for production of IFN- $\gamma$ or IL-2 by intracellular staining and flow cytometry. Cells were stimulated with PMA/ionomycin for 6 hours in the presence of GolgiPlug (BD Biosciences) or treated with HIV-1 clade B Env, Gag, Pol peptide pool (from NIH AIDS Reagent Program, www.aidsreagent.org) overnight and an additional 6 hours of GolgiPlug treatment. Cells were then stained with CD45, CD3, CD8, IF N- $\gamma$, and IL-2 (eBioscience).

ART treatment and IFNR blockade treatment. For ART, HIV-infected mice were treated by daily injection of $500 \mu \mathrm{l}$ of TDF $(8.75 \mathrm{mg} / \mathrm{kg}) /$ FTC $(13 \mathrm{mg} / \mathrm{kg}) /$ raltegravir $(17.5 \mathrm{mg} / \mathrm{kg})$ dissolved in PBS. For IFNR blockade treatment, HIV-infected mice, either untreated or treated with ART, were treated with $100 \mu \mathrm{g}$ anti-IFNR2 blocking antibody (clone MMHAR-2) or an IgG isotype control (eBioscience) once every 2 days by i.p. injection for 1 week. One hundred micrograms every 2 days was chosen because it was the minimum dosage to maintain lower ISG expression in the humanized BLT mice (data not shown). A treatment course of 1 week was chosen because it has been shown to effectively target IFN-I signaling in LCMV-infected mice (5). For mice that were treated with both ART and IFNR blockade, the anti-IFNR2 antibody was given concurrently with the ART regiment at the last week of ART treatment.

Real-time PCR. To measure the levels of cell-associated HIV RNA and the ISGs MX1 and OAS1, with HPRT1 as an internal control, splenocytes and peripheral blood mononuclear cells were harvested for RNA extraction and making of cDNA using the High-Capacity cDNA 
A

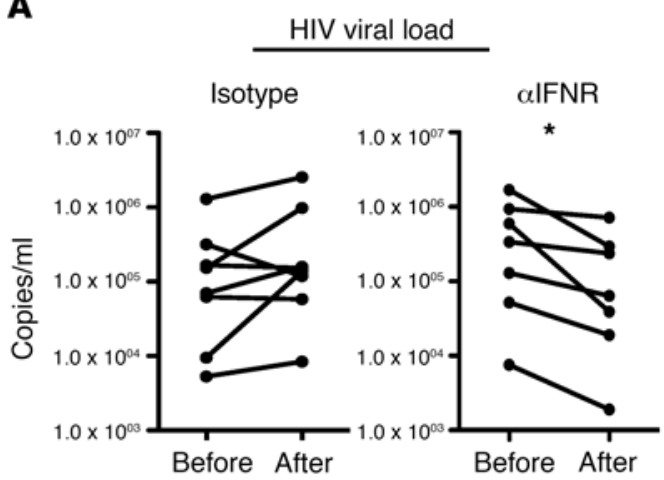

B

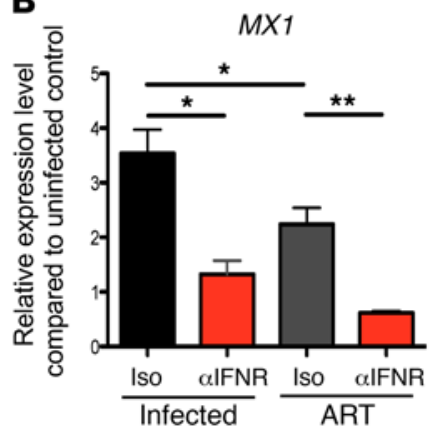

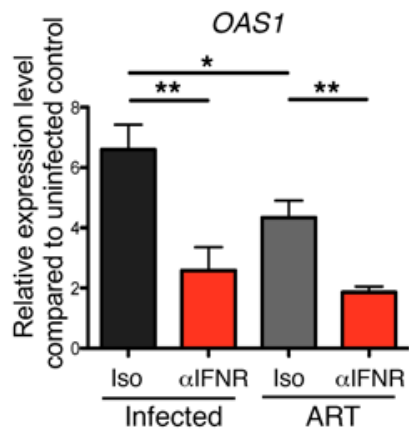

C

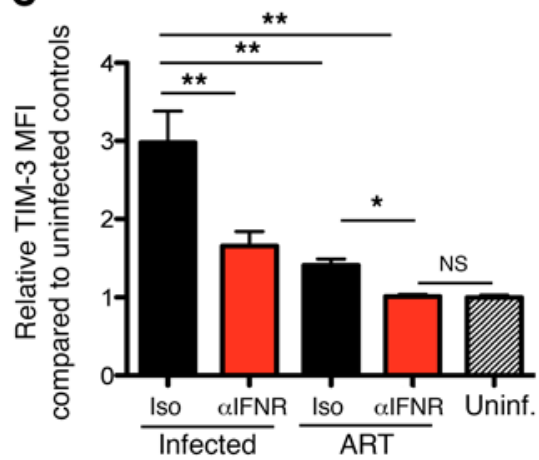

E

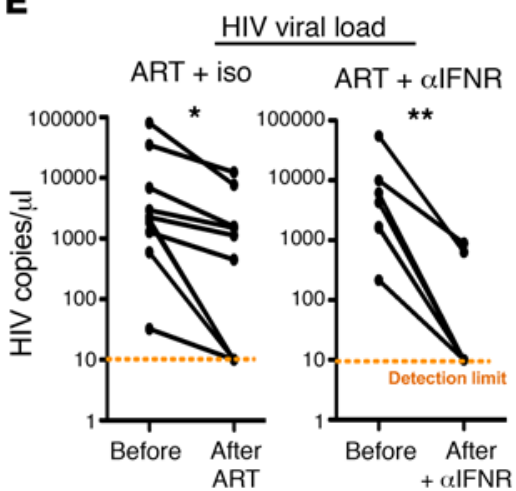

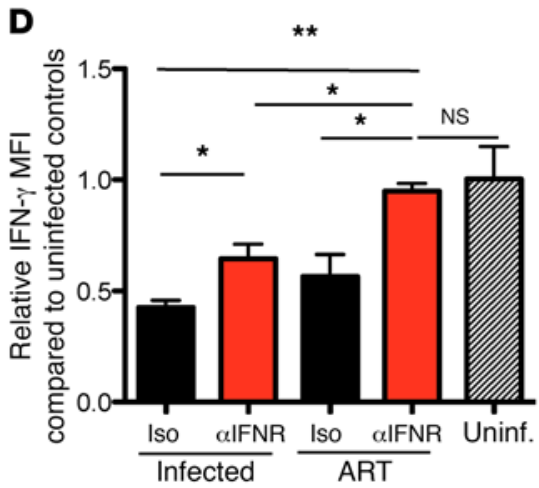

$\mathbf{F}$

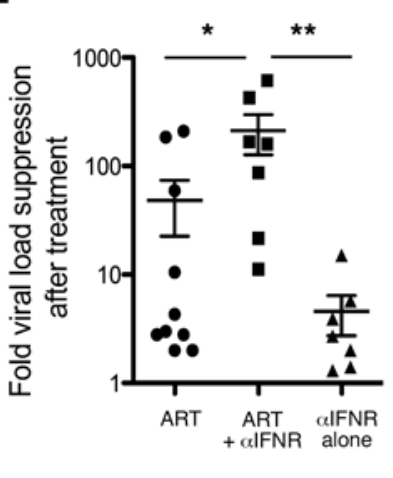

G

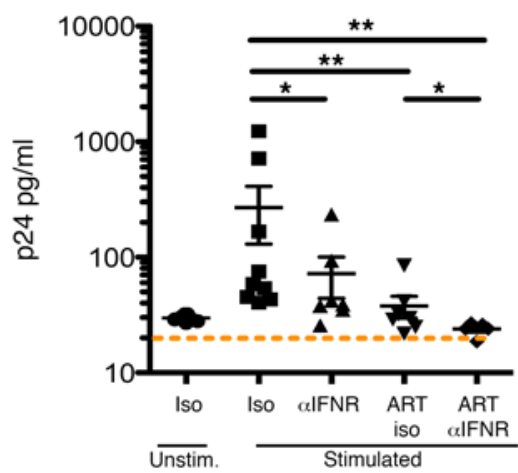

Figure 4. IFNR blockade treatment reduces viral load, and combination of ART and IFNR blockade promotes faster viral suppression and reduces viral reservoir. (A) Changes of plasma viral load before and after isotype treatment or IFNR blockade. (B) MX1 and OAS1 expression levels from PBMCs in infected mice in different treatment groups as measured by real-time RT-PCR ( $n=5-11$ per group). (C) Relative TIM-3 expression level of CD8 ${ }^{+}$T cells in PBMCs from infected mice in different treatment groups as compared with uninfected mice ( $n=5-11$ per group). (D) Relative IFN- $\gamma$ MFI of PMA/ionomycin-stimulated $\mathrm{CD}^{+} \mathrm{T}$ cells from infected mice in different treatment groups as compared with uninfected mice ( $n=5-11$ per group). (E) Changes of viral loads in HIV-1infected NSG-BLT mice that were treated with ART or ART plus IFNR blockade ( $n=7-10$ per group). (F) Fold viral suppression after ART treatment, ART with IFNR blockade, or IFNR blockade alone ( $n=7-10$ per group). (C) HIV p24 production in the supernatant (as measured by ELISA collected from stimulated, sorted HSA- cells following the indicated treatments; $n=4-9$ per group). The Mann-Whitney $U$ test was used to compare 2 groups ( ${ }^{*} P<0.05,{ }^{*} P<0.005$ ), and the Kruskal-Wallis test was used for multiple comparisons (B-D, F, and $\mathbf{G}$; all have $P<0.05$ ). Data represent mean \pm SEM.

Reverse Transcription Kit (Thermo Fisher Scientific). Real-time PCR was performed using the following primers and probes: HIV-1 forward primer: 5'-CAATGGCAGCAATTTCACCA-3'; HIV-1 reverse primer: 5'-GAATGCCAAATTCCTGCTTGA-3'; HIV-1 probe: 5'-[6-FAM] CCCACCAACAGGCGGCCTTAACTG[Tamra-Q]-3'; Single Tube TaqMan Gene Expression Assays: human HPRT1 (Hs01003267_m1), MX1 (Hs00895608_m1), and OAS1 (Hs00973635_m1).

Plasma viral load. To measure HIV plasma viral load, blood was collected from mice via retro-orbital bleeding or heart puncture at end point. Blood was then spun at $1,200 \mathrm{~g}$ to collect plasma supernatant. Viral RNA was extracted using QIAamp Viral RNA Mini Kit (Qiagen), and 1-step real-time PCR was performed using TaqMan RNA-To-Ct 1-Step Kit (Thermo Fisher Scientific) with the following primers and probe: HIV-1 forward primer: 5'-CAATGGCAGCAATTTCACCA-3'; HIV-1 reverse primer: 5'-GAATGCCAAATTCCTGCTTGA-3'; HIV-1 probe: 5'-[6-FAM]CCCACCAACAGGCGGCCTTAACTG[Tamra-Q]-3'.

Assay for reactivatable virus. The assay for reactivatable virus was performed similarly to what was previously reported $(15,19)$. In short, 
mice were infected with $\mathrm{HIV}_{\mathrm{NL4}-3 \mathrm{HSA}}$, which will allow actively infected cells to express mouse heat stable antigen (HSA). After the mice were sacrificed, splenocytes were isolated, and $\mathrm{HSA}^{+}$cells were depleted using biotin-conjugated anti-HSA antibody (eBioscience) and Streptavidin Microbeads (Miltenyi Biotec). HSA ${ }^{-}$cells were then activated with anti-CD3 (plate bound) and anti-CD28 (soluble) and indinavir for 3 days. Supernatant from activated cells was collected and purified for HIV p24 antigen ELISA assay, and cells were collected for HIV DNA and RNA real-time PCR analysis.

Statistics. A total of 5 independent cohorts of mice $(n=15-30$ total mice, each constructed from the same donor tissues) were used in this study for various experiments. Two cohorts were pooled for comparing uninfected and infected mice. Three cohorts were pooled for comparing uninfected mice versus infected mice treated with isotype antibody or IFNR blockade versus infected mice treated with ART and isotype antibody or IFNR blockade. To pool mice from different cohorts, each measurement (ISG expression level or MFI of activation/exhaustion markers) was first normalized to the uninfected controls of the cohort. Each analysis, including those shown in bar graphs such as the MFI comparison or real-time PCR, contains at least 4-7 biological replicates (number of mice). For flow analysis of CD4 and CD8 cells, at least 1,000 events were acquired for the population of interest. For analysis of data that contains more than 2 groups (depicted in Figure 1C; Figure 3, A and B; and Figure 4, B-D, F, and G), the Kruskal-Wallis test was performed to compare samples; and all of these data have $P$ value less than 0.05 . To compare statistical difference between 2 groups, Mann-Whitney $U$ tests were used. $P$ values less than 0.05 by Kruskal-Wallis or Mann-Whitney were considered significant. Consultation on statistical analysis was performed with the UCLA Center for AIDS Research Biostatistics Core and the UCLA Biostatistics Department.

Study approval. Peripheral blood mononuclear cells were obtained at UCLA in accordance with UCLA IRB-approved protocols under written informed consent using an IRB-approved written consent form by the UCLA Center for AIDS Research Virology Laboratory and distributed for this study without personal identifying information. Human fetal tissue was purchased from Advanced Biosciences Resources or from StemExpress and was obtained without identifying information and did not require IRB approval for its use. Animal research described in this article was performed under the written approval of the UCLA Animal Research Committee in accordance with all federal, state, and local guidelines. All surgeries were performed under ketamine/xylazine and isoflurane anesthesia, and all efforts were made to minimize animal pain and discomfort.

\section{Author contributions}

AZ designed the experiments. AZ, VR, CY, BL, NC, JR, MC, HM, $\mathrm{SK}, \mathrm{PS}$, and NR performed the experiments. AZ analyzed the data. $\mathrm{AZ}, \mathrm{DGB}$, and SGK wrote the manuscript.

\section{Acknowledgments}

We thank Jerome Zack and Matthew Marsden for provision of ART drugs and for helpful discussions. This work was funded by NIH grants AI078806 and AI110306-01 (to SGK) and AI085043 (to DGB); the UCLA Center for AIDS Research (P30AI28697); the California Center for Regenerative Medicine (TR4-06845); the UC Multicampus Research Programs and Initiatives, California Center for Antiviral Drug Discovery, and California HIV/AIDS Research Program (F12-LA-215 to AZ); NIH grant T32-AI060567 (to AZ); and the UCLA AIDS Institute and UCLA Center for AIDS Research (AI28697 to AZ). The flow cytometry machine used in the study was purchased through a Pendleton Foundation grant.

Address correspondence to: Scott G. Kitchen, UCLA AIDS Institute, 615 Charles E. Young Drive South, Los Angeles, California 90095, USA. Phone: 310.983.1251; E-mail: skitchen@ucla.edu. Or to: David G. Brooks, Princess Margaret Cancer Center, 610 University Avenue, Toronto, Ontario M5G2M9, Canada. Phone: 416.634.7238; E-mail: dbrooks@uhnresearch.ca.
1. Jones RB, et al. Tim-3 expression defines a novel population of dysfunctional T cells with highly elevated frequencies in progressive HIV-1 infection. J Exp Med. 2008;205(12):2763-2779.

2. McMichael AJ, Rowland-Jones SL. Cellular immune responses to HIV. Nature. 2001;410(6831):980-987.

3. Jin HT, et al. Cooperation of Tim-3 and PD-1 in CD8 T-cell exhaustion during chronic viral infection. Proc Natl Acad Sci U S A. 2010;107(33):14733-14738.

4. Khaitan A, Unutmaz D. Revisiting immune exhaustion during HIV infection. Curr HIV/AIDS Rep. 2011;8(1):4-11.

5. Wilson EB, et al. Blockade of chronic type I interferon signaling to control persistent LCMV infection. Science. 2013;340(6129):202-207.

6. Ng CT, Snell LM, Brooks DG, Oldstone MB. Networking at the level of host immunity: immune cell interactions during persistent viral infections. Cell Host Microbe. 2013;13(6):652-664.

7. Jacquelin B, et al. Nonpathogenic SIV infection of African green monkeys induces a strong but rapidly controlled type I IFN response. J Clin Invest. 2009;119(12):3544-3555.
8. Wherry EJ, Kurachi M. Molecular and cellular insights into T cell exhaustion. Nat Rev Immunol. 2015;15(8):486-499.

9. Denton PW, et al. Generation of HIV latency in humanized BLT mice. J Virol. 2012;86(1):630-634

10. Wilson EB, Brooks DG. Decoding the complexity of type I interferon to treat persistent viral infections. Trends Microbiol. 2013;21(12):634-640.

11. Deng K, et al. Broad CTL response is required to clear latent HIV-1 due to dominance of escape mutations. Nature. 2015;517(7534):381-385.

12. Hazenberg MD, et al. Persistent immune activation in HIV-1 infection is associated with progression to AIDS. AIDS. 2003;17(13):1881-1888.

13. Rajasuriar R, Khoury G, Kamarulzaman A, French MA, Cameron PU, Lewin SR. Persistent immune activation in chronic HIV infection: do any interventions work? AIDS. 2013;27(8):1199-1208.

14. Sauce D, Elbim C, Appay V. Monitoring cellular immune markers in HIV infection: from activation to exhaustion. Curr Opin HIV AIDS. 2013;8(2):125-131.

15. Marsden MD, et al. HIV latency in the humanized BLT mouse. J Virol. 2012;86(1):339-347.

16. Brooks DG, Arlen PA, Gao L, Kitchen CM, Zack
JA. Identification of T cell-signaling pathways that stimulate latent HIV in primary cells. Proc Natl Acad Sci U S A. 2003;100(22):12955-12960.

17. Teijaro JR, et al. Persistent LCMV infection is controlled by blockade of type I interferon signaling. Science. 2013;340(6129):207-211.

18. Sandler NG, et al. Type I interferon responses in rhesus macaques prevent SIV infection and slow disease progression. Nature. 2014;511(7511):601-605.

19. Brooks DG, Kitchen SG, Kitchen CM, ScriptureAdams DD, Zack JA. Generation of HIV latency during thymopoiesis. Nat Med. 2001;7(4):459-464.

20. Azzoni L, et al. Pegylated interferon alfa-2a monotherapy results in suppression of HIV type 1 replication and decreased cell-associated HIV DNA integration. JInfect Dis. 2013;207(2):213-222.

21. Asmuth DM, et al. Safety, tolerability, and mechanisms of antiretroviral activity of pegylated interferon alfa-2a in HIV-1-monoinfected participants: a phase II clinical trial. JInfect Dis. 2010;201(11):1686-1696.

22. Bruner KM, et al. Defective proviruses rapidly accumulate during acute HIV-1 infection. Nat Med. 2016;22(9):1043-1049.

23. Acchioni C, Marsili G, Perrotti E, Remoli AL, 
Sgarbanti M, Battistini A. Type I IFN - a blunt spear in fighting HIV-1 infection. Cytokine Growth Factor Rev. 2015;26(2):143-158.

24. Larsson M, et al. Molecular signatures of T-cell inhibition in HIV-1 infection. Retrovirology. 2013;10:31.

25. Rodríguez-García M, et al. Expression of PD-L1 and PD-L2 on human macrophages is upregulated by HIV- 1 and differentially modulated by IL-10. J Leukoc Biol. 2011;89(4):507-515.

26. Brainard DM, et al. Induction of robust cellular and humoral virus-specific adaptive immune responses in human immunodeficiency virus-infected humanized BLT mice. J Virol. 2009;83(14):7305-7321.

27. Planès R, BenMohamed L, Leghmari K, Delobel P, Izopet J, Bahraoui E. HIV-1 Tat protein induces PD-L1 (B7-H1) expression on dendritic cells through tumor necrosis factor $\alpha$ - and tolllike receptor 4-mediated mechanisms. J Virol. 2014;88(12):6672-6689.

28. Dudek TE, et al. Rapid evolution of HIV-1 to functional $\mathrm{CD}^{+} \mathrm{T}$ cell responses in humanized BLT mice. Sci Transl Med. 2012;4(143):143ra98.
29. Kitchen SG, et al. Engineering antigen-specific T cells from genetically modified human hematopoietic stem cells in immunodeficient mice. PLOS One. 2009;4(12):e8208.

30. Kitchen SG, et al. In vivo suppression of HIV by antigen specific $T$ cells derived from engineered hematopoietic stem cells. PLoS Pathog. 2012;8(4):e1002649.

31. Goujon C, Malim MH. Characterization of the alpha interferon-induced postentry block to HIV-1 infection in primary human macrophages and T cells. JVirol. 2010;84(18):9254-9266. 